\title{
Sistema visual humano: evidência psicofísica para filtros de freqüência angular baixa
}

\author{
Natanael Antonio dos Santos \\ Universidade Federal da Paraíba \\ Maria Lúcia de Bustamante Simas \\ Universidade Federal de Pernambuco \\ Renata Maria Toscano Barreto Lyra Nogueira \\ Universidade Federal da Paraíba
}

\begin{abstract}
Resumo
O objetivo deste trabalho foi mensurar curvas de resposta ao contraste para os filtros de freqüências angulares de banda estreita de 1, 2, 3 e 4 ciclos $/ 360^{\circ}$. Foram estimadas nove curvas para cada filtro com o método psicofísico de somação de resposta de supralimiar aliado ao método da escolha forçada. Tomaram parte neste experimento cinco participantes adultos com acuidade visual normal ou corrigida. Os resultados demonstraram somações máximas de limiar de contraste na freqüência de teste dos filtros de 1, 2, 3 e 4 ciclos $/ 360^{\circ}$ circundadas por inibições nas freqüências vizinhas às freqüências angulares de teste de cada filtro. Estes resultados são consistentes com a existência de filtros de freqüências angulares de banda estreita operando no sistema visual humano através do processo de somação ou inibição na faixa de freqüências angular baixa.
\end{abstract}

Palavras-chave: percepção visual da forma; filtro angular; freqüência angular; somação de supralimiar

\begin{abstract}
Human visual system: psychophysical evidence for low angular frequency filters. The aim of this work was to measure narrow-band frequency response curves for four angular frequency filters whose test frequencies were $1,2,3$ and 4 cycles $/ 360^{\circ}$. Five human observers with normal or corrected visual acuity measured nine curves for each filter with a supra-threshold response summation psychophysical method allied within a forced-choice method. The results showed maximum summation effects at test frequency for filters 1, 2, 3 and 4 cycles $/ 360^{\circ}$ surrounded on both sides by strong inhibition. These results are consistent with the existence of narrow-band angular frequency filters operating in human visual system either through summation or inhibition of specific low angular frequency ranges.
\end{abstract}

Keywords: visual perception of form; angular frequency filter; angular frequency; supra-threshold summation method

$\mathrm{E}$ ntender como o sistema visual humano (SVH) reconhece objetos ou cenas visuais é um processo fundamental na percepção ou no processamento visual da forma. A idéia da decomposição de objetos complexos em subunidades simples ou elementares e o fato de unidades ou células nervosas responderem apenas a determinadas características dos objetos levaram vários pesquisadores a caracterizar a resposta do sistema visual para estímulos elementares com o objetivo de tentar identificar os atributos ou as subunidades nas quais um padrão complexo é decomposto e processado.

Uma das principais abordagens utilizada para investigar o processamento ou a percepção visual da forma em humanos se baseia no modelo de canais múltiplos de freqüências espaciais. Esta abordagem proposta originalmente por
Campbell e Robson (1968) assume que o processamento ou a percepção visual de objetos ocorre porque o nosso sistema visual executa uma análise ou decomposição desse padrão complexo em seus componentes mais elementares, uma espécie de análise de Fourier (Blakemore \& Campbell, 1969a; 1969b; Campbell \& Robson, 1968; R. L. De Valois \& K. K. De Valois, 1988; Sachs, Nachmias, \& Robson, 1971). Em outras palavras, as teorias de canais múltiplos se fundamentam no princípio de que durante a percepção visual da forma, uma cena visual qualquer é decomposta em seus componentes mais elementares (freqüências espaciais), os quais o córtex visual utiliza para fazer suas representações (Blakemore \& Campbell, 1969b; R. L. De Valois \& K. K. De Valois, 1988).

As primeiras evidências psicofísicas para canais múltiplos de freqüências espaciais vieram a partir do surgimento 
da técnica da adaptação seletiva a freqüências espaciais. Essa técnica surgiu de um trabalho de Gilinski (1968), que observou que depois de visualizar um padrão listrado de contraste alto por um período de tempo, observadores podiam mostrar um prejuízo para perceber padrões de freqüência e orientação idênticas, mas de contraste mais baixo. Esse fenômeno descrito por Gilinski se tornou conhecido como adaptação e foi observado ser altamente seletivo para orientação e freqüência espacial (Blakemore \& Campbell, 1969a; K. L. De Valois \& K. K. De Valois, 1988; Menees, 1998). Contraste é definido pela relação entre a diferença da luminância máxima (listra clara) e luminância mínima (listra escura) dividida pela soma da luminância máxima e mínima.

Um dos primeiros e mais convincentes estudos realizados com o método da adaptação seletiva favorável à existência de canais múltiplos de freqüências espaciais foi realizado por Blakemore e Campbell (1969a). Esses autores verificaram que a adaptação à grade senoidal eleva o limiar da grade senoidal próxima à freqüência adaptada.

Atualmente, estudos psicofísicos em humanos e estudos neurofisiológicos em macacos e gatos fornecem fortes evidências para o modelo de canais múltiplos ou mecanismos sintonizados para banda estreita de freqüências espaciais (Blakemore \& Campbell, 1969a, 1969b; R. L. De Valois \& K. K. De Valois, 1988; Dakin \& Hess, 1997; Maffei \& Fiorentini, 1973; Menees, 1998; Olds \& Engel, 1998; Santos \& Simas, 2002; Santos, Simas, \& Nogueira, 2004; Simas \& Santos, 2002a; 2002b; Wilson, Levi, Maffei, Rovamo, \& De Valois, 1990). Entretanto, a maioria absoluta destes utiliza grade senoidal vertical em coordenadas cartesianas para caracterizar a existência de filtros ou canais sintonizados para freqüências espaciais. Freqüência espacial é o número de ciclos (ou listras claras e escuras) por grau de ângulo visual (cpg). Um canal do ponto de vista psicofísico seria, por exemplo, um filtro com estrutura independente no sistema, que lida com uma faixa estreita de freqüência espacial. Já do ponto de vista fisiológico, um canal significa uma população neuronal sintonizada para uma banda-estreita de freqüência espacial (Santos \& Simas, 2001b, Santos \& Simas, 2002).

\section{Filtragens de freqüências radiais e angulares pelo sistema visual humano}

Existem evidências, baseadas em estudos psicofísicos e neurofisiológicos, de que o processamento linear ou filtragem espacial local sintonizada para orientação e freqüência espacial de estímulos em coordenadas cartesianas projetados na retina ocorre nos estágios iniciais da visão, isto é, em neurônios do córtex visual primário, V1 (K. L. De Valois \& K. K. De Valois, 1988; Manahilov \& Simpson, 2001; Wilkinson et al., 2000; Wilson \& Wilkinson, 1998; Wilson, Wilkinson, \& Asaad, 1997). Por outro lado, até o momento não encontramos nenhum relato de que unidades neurais da área visual primária, V1, executem filtragem de estímulos em coordenadas polares, amostrando áreas grandes do campo visual. Neste contexto, resultados psicofísicos em humanos (Wilkinson, Wilson \& Habak, 1998; Wilson \& Wilkinson, 1997; 1998; Wilson et al., 1997), neurofisiológicos em primatas
(Bruce, Desimone, \& Gross, 1981; Desimone, 1991; Desimone \& Schein, 1987, Gallant, Cannor, Rakshit, Lewis, \& van Essen, 1996) e imagem de ressonância magnética funcional em humanos (Wilkinson et al., 2000) destacam que o processamento da informação que ocorre em vias intermediárias (e.g., área visual V4) e áreas mais avançadas (e.g., córtex visual ínferotemporal, IT) do sistema visual são modeladas por filtros espaciais globais em coordenadas polares. Alguns desses trabalhos destacam que a área V4 pode formar o principal estágio intermediário da visão da forma de V1 para IT (Heywood, Gadotti, \& Cowey, 1992; Merigan, 1996; van Essen, Anderson, \& Felleman, 1992; Wilkinson et al., 2000; Young, 1992). Assim, é possível que a filtragem e integração de padrões radiais e angulares em coordenadas polares ocorram em áreas visuais extra-estriatais (e.g., V4 e IT). O estudo de Wilkinson et al. (2000), com a técnica de imagem de ressonância magnética funcional em humanos, reforçou os estudos psicofísicos com humanos e neurofisiológicos com primatas que demonstraram inicialmente que estímulos em coordenadas polares são processados em áreas visuais extra-estriatais (e.g., V4 e IT).

Neste sentido, estudos psicofísicos têm procurado investigar a idéia de canais ou mecanismos distintos e seletivos para freqüências radiais e angulares em coordenadas polares (Santos \& Simas, 2002; Simas \& Dodwell, 1990; Simas, Frutuoso, \& Vieira, 1992; Simas \& Santos, 2002a; 2002b). Por exemplo, Santos e Simas (2002) mensuram as curvas de resposta ao contraste para os filtros de freqüências radiais de banda-estreita com contraste máximo centrado em 1 e 4 cpg. Os resultados encontrados com o método psicofísico de somação de resposta de supralimiar aliado ao método da escolha forçada demonstraram somações máximas de limiar de contraste na freqüência de teste dos filtros de 1 e 4 cpg circundadas por inibições nas freqüências vizinhas às freqüências radiais de teste de cada filtro. Estes resultados foram favoráveis à existência de filtros de freqüências radiais de banda-estreita operando no sistema visual humano através do processo de somação ou inibição na faixa de freqüência estudada.

Por outro lado, Simas e Dodwell (1990) mensuraram a resposta do SVH para o filtro de freqüência angular de 24 ciclos. Posteriormente, Simas e colaboradores estimaram a resposta do SVH para um conjunto de sete filtros de freqüências angulares (p.ex., os filtros angulares de 2, 4, 9, 13, 16, 24 e 47 ciclos $/ 360^{\circ}$ ) (Simas et al., 1992, Simas \& Santos, 2002b). Todos os estudos foram realizados com o método psicofísico de somação de resposta de supralimiar aliado ao método da escolha forçada. Os resultados encontrados por Simas e colaboradores demonstraram efeitos de somação absoluta e relativa nas freqüências angulares de teste dos filtros circundadas por inibições, o que levou os autores a concluírem a favor da existência de alguma seletividade para faixas específicas de freqüências angulares.

O método psicofísico de somação de resposta de supralimiar é uma adaptação do método psicofísico de somação de resposta de subliminar de Kulikowski e King Smith (1973). No estudo original os referidos autores empregaram metade do contraste necessário para alcançar o limiar de contraste dos respectivos estímulos de teste e de fundo 
(ruído). A finalidade foi obter, eventualmente, a somação do contraste da freqüência de teste com o contraste da freqüência de fundo e alcançar, desse modo, o limiar de detecção. Nos experimentos com o método de somação de resposta de supralimiar, em vez de se utilizar somação de resposta com níveis de contraste sublimiar para mensurar o máximo da função, utiliza-se somação de resposta de supralimiar de contraste, sendo a freqüência de teste somada pixel a pixel às freqüências de fundo que são apresentadas a alto contraste. Nesse, enquanto o contraste da freqüência de teste é variado de acordo com a sensibilidade do voluntário, o contraste da freqüência de fundo é fixo acima do limiar (isto é, supralimiar). Dessa forma, se um estímulo contendo apenas uma das freqüências de fundo é comparado a um estímulo em que a mesma freqüência de fundo é somada a freqüência de teste, a única forma de diferenciar entre os dois estímulos é detectar a presença da freqüência de teste em um destes. Informações mais detalhadas sobre este método podem ser encontradas na literatura (Santos \& Simas, 2002; Simas \& Dodwell, 1990; Simas et al., 1992; Simas \& Santos, 2002b).

A utilização do método psicofísico de somação de resposta de supralimiar possibilita três tipos de respostas pelo sistema visual humano: (1) aquelas em que determinadas freqüências de fundo podem se somar à freqüência de teste do filtro facilitando a sua detecção (efeito de somação); (2) aquelas em que as freqüências de fundo e de teste podem não se somar, não facilitando nem dificultando sua detecção (efeito independente); ou, (3) aquelas em que as freqüências de fundo podem inibir a percepção da freqüência de teste do filtro, dificultando a sua detecção (efeito inibitório). A sensibilidade ao contraste da freqüência de teste em função das freqüências de fundo, usando como referência o limiar absoluto de detecção da freqüência de teste, indicará a independência dos mecanismos de detecção ou os efeitos de somação ou inibição.

Este trabalho dá continuidade aos experimentos que procuram caracterizar a resposta do SVH para padrões espaciais em coordenadas polares. Boa parte destes experimentos se baseia na possibilidade das áreas superiores do sistema visual (e.g., V4 e IT) processarem informação visual de forma em termos de freqüências radiais e angulares, o que pode envolver filtragem de faixas ou áreas inteiras do campo visual. Neste sentido, o presente estudo procurou caracterizar mecanismos ou filtros putativos com contraste máximo centrados nas freqüências angulares de 1, 2, 3 e 4 ciclos $/ 360^{\circ}$, utilizando o método psicofísico de somação de resposta de supralimiar. O objetivo principal foi estabelecer a existência de filtros de freqüências angulares de banda estreita em coordenadas polares na faixa de baixa freqüência.

\section{Método}

\section{Participantes}

Participaram dos experimentos cinco adultos de ambos os sexos com acuidade visual normal ou corrigida na faixa etária de 20-25 anos. A participação foi voluntária mediante assinatura de termo de consentimento livre e esclarecido.

\section{Equipamentos e estímulos}

Foi utilizado um sistema que compreende um monitor de vídeo SONY-BVM-1910, com entrada RGBsync entrelaçada e interfaciado a um microcomputador 486 de $30 \mathrm{MHz}$ através de uma placa de aquisição Data-Translation DT-2853. Um programa escrito em linguagem $C$ foi desenvolvido para executar os experimentos. Uma cadeira giratória foi fixada a $150 \mathrm{~cm}$ da tela do monitor de vídeo. Uma tábua cinza foi situada acima do monitor de vídeo para a qual o voluntário deveria fixar o olhar, entre uma apresentação e outra, com o objetivo de diminuir os efeitos de pós-imagem. A luminância média foi de $6,9 \mathrm{~cd} / \mathrm{m}^{2}$ ajustada por um fotômetro do tipo Asahi Pentax Spotmeter, com precisão de um grau. A luminância mínima e máxima da tela foi de $6,2 \mathrm{~cd} / \mathrm{m}^{2}$ e de $7,5 \mathrm{~cd} / \mathrm{m}^{2}$, respectivamente. O ambiente do laboratório era cinza para melhor controle da luminância.

Os estímulos de freqüências angulares foram gerados em tons de cinza e apresentados em tempo real no monitor. Todos os estímulos também eram circulares com um diâmetro de 7,25 graus de ângulo visual a $150 \mathrm{~cm}$ de distância da tela (Figura 1).

As estimativas foram feitas utilizando pares de estímulos, estímulo de teste e estímulo de fundo. O estímulo de teste era composto pela freqüência de teste do filtro (1, 2, 3 e 4 ciclos $/ 360^{\circ}$ ) somada a uma das freqüências de fundo, por exemplo: 1, 2, 3, 4, 5, 6, 7, 8, 9, 10, 12, 16, 24, 32, 48, 64 ou 96 ciclos/ $360^{\circ}$. O estímulo de fundo foi a própria freqüência de fundo sozinha. A Figura 1 ilustra exemplos de pares de estímulos de freqüências angulares utilizados, por exemplo, para mensurar o filtro angular de 3 ciclos $/ 360^{\circ}$.

\section{Procedimento}

O procedimento para medir cada filtro angular utilizando o método de somação de resposta de supralimiar aliado ao método da escolha forçada consistiu na apresentação sucessiva simples do par de estímulos; e o voluntário tinha de escolher dentre os dois estímulos qual continha a freqüência angular de teste do filtro somada à freqüência angular de fundo. O contraste da freqüência de fundo foi mantido constante e correspondia a cinco vezes o limiar absoluto de detecção da freqüência de teste do filtro angular (F1, F2, F3 e F4), tanto no estímulo de teste como no estímulo de fundo. Somente o contraste supralimiar da freqüência de teste era variado, diminuindo ou aumentando de acordo com o método da escolha forçada (Santos \& Simas, 2002; Santos, Simas, \& Nogueira, 2004; Wetherill \& Levitt, 1965). O critério adotado para variar o contraste da freqüência de teste era o de três acertos consecutivos para decrescer uma unidade e um erro para acrescer da mesma unidade (0,08\%). Este procedimento experimental se baseia no cálculo da probabilidade de acertos consecutivos por parte do voluntário, ou seja, em cerca de 100 apresentações de escolhas entre os dois estímulos, o estímulo de teste é percebido 79\% das vezes pelo voluntário.

A seqüência temporal foi iniciada com um sinal sonoro seguido imediatamente pela apresentação do primeiro estí- 

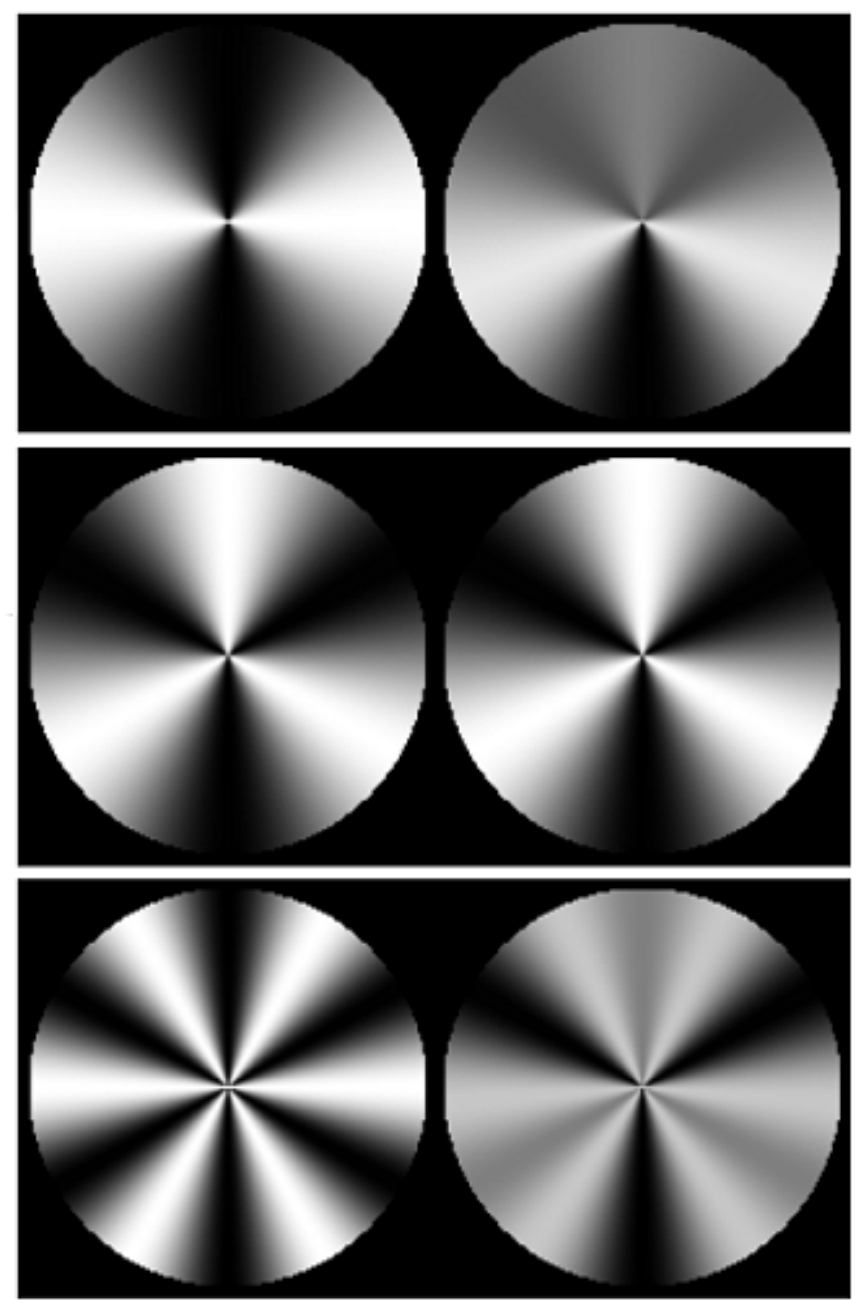

Figura 1. Exemplos de pares de estímulos de freqüências angulares utilizados para estimar o filtro angular de 3 ciclos. Acima e à esquerda, a freqüência de 2 ciclos e, à direita, 2 ciclos somada à frequiência de 3 ciclos; ao centro e à esquerda, 3 ciclos e, à direita, 3 ciclos somada à de 3 ciclos; e embaixo e à esquerda, 6 ciclos e, à direita, 6 ciclos somada à freqüência de 3 ciclos.

mulo por $2 \mathrm{~s}$, seguido de um intervalo entre estímulos de $1 \mathrm{~s}$, seguido pela apresentação do segundo estímulo por 2 s e da resposta do participante. A ordem de apresentação dos estímulos era aleatória. Se a resposta do participante fosse correta, ela era seguida por outro sinal sonoro e um intervalo de 3 s para a seqüência temporal se repetir. Em outras palavras, $3 \mathrm{~s}$ era o intervalo entre tentativas, independentemente da resposta (ou escolha) ser correta ou não. O sinal sonoro que indicava o início da apresentação do par de estímulos e o que indicava a escolha correta eram diferentes. A sessão experimental variava em duração dependendo dos erros e acertos do participante até proporcionarem um total de 10 máximos e 10 mínimos conforme requerido para o final automático da mesma. Sua duração era da ordem de 10-20 min.

Os participantes foram orientados antes da sessão a pressionar a barra de espaço quando julgassem que o estímulo de teste tivesse sido apresentado primeiro e qualquer tecla acima da barra de espaço quando julgasse que o mesmo tivesse sido apresentado em segundo lugar, isto é, após o estímulo de fundo.

A ordem de mensuração das freqüências angulares para cada curva foi aleatorizada por sorteio pelo voluntário antes de mensurar cada uma das três curvas para cada um dos quatro filtros. Todas as estimativas foram feitas à distância de $150 \mathrm{~cm}$, com visão binocular.

Uma curva de filtragem era composta por 17 pontos ou freqüências e cada freqüência correspondeu a uma sessão experimental. Assim, as 17 sessões experimentais requeridas para medir cada um dos quatro filtros (F1, F2, F3 e F4) foi realizada pelo menos três vezes, em dias diferentes, por pelo menos três participantes. No geral, um total de nove curvas (ou funções) foi medida para cada filtro gerando uma amostra de 180 valores para cada um dos 17 pontos estimados. 


\section{Resultados}

A Figura 2 mostra as curvas de respostas ao contraste para os filtros de freqüências angulares 1 (F1), 2 (F2), 3 (F3) e 4 (F4) ciclos $/ 360^{\circ}$. Cada curva de filtragem representa os limiares de contraste médios obtidos para cada filtro estimado. As freqüências angulares de fundo (abscissa) são apresentadas nos gráficos em função da quantidade de contraste necessário (ordenada), para identificar a presença da freqüência angular de teste somada a cada freqüência angular de fundo. Estamos chamando este dado de limiar para a freqüência angular de teste somada a cada freqüência angular de fundo de limiar de contraste para o estímulo teste na soma. As barras verticais indicam os erros padrões das médias. A linha horizontal representa a linha de base (LB), que equivale ao limiar absoluto para detectar a freqüência angular de teste do filtro (1, 2, 3 e 4 ciclos $/ 360^{\circ}$ ) isolada.

Nosso tratamento estatístico foi estimar o erro padrão da média para cada distribuição de 180 valores mensurados para cada ponto e corrigidos para o tamanho da amostra pelo estatístico t-Student, para obter o nível de confiança de 99\%. Mesmo quando os intervalos dos erros padrões da média assim corrigidos se superpõem até pela metade, um teste $t$ Student para amostras correlacionadas revela diferenças entre as médias que são significativas com $p<0,05$. Nos casos em que os erros barras não se sobrepõem, as médias são significativas com $p<0,001$. A ANOVA tende a mostrar interações e efeitos significativos em todos fatores e não acrescenta muita informação.

Os filtros de freqüências angulares F1, F2, F3 e F4 apresentam efeito de somação máxima em suas respectivas freqüências angulares de teste circundadas de inibição nas freqüências vizinhas às freqüências angulares de teste (ver Figura 2). O efeito de somação (ou filtragem) mostra que o limiar de detecção de contraste do SVH é menor para freqüência de teste somada a ela mesma do que o limiar de contraste absoluto da freqüência teste sozinha. A sensibilidade ao contraste da freqüência de teste em função das freqüências de fundo, usan-
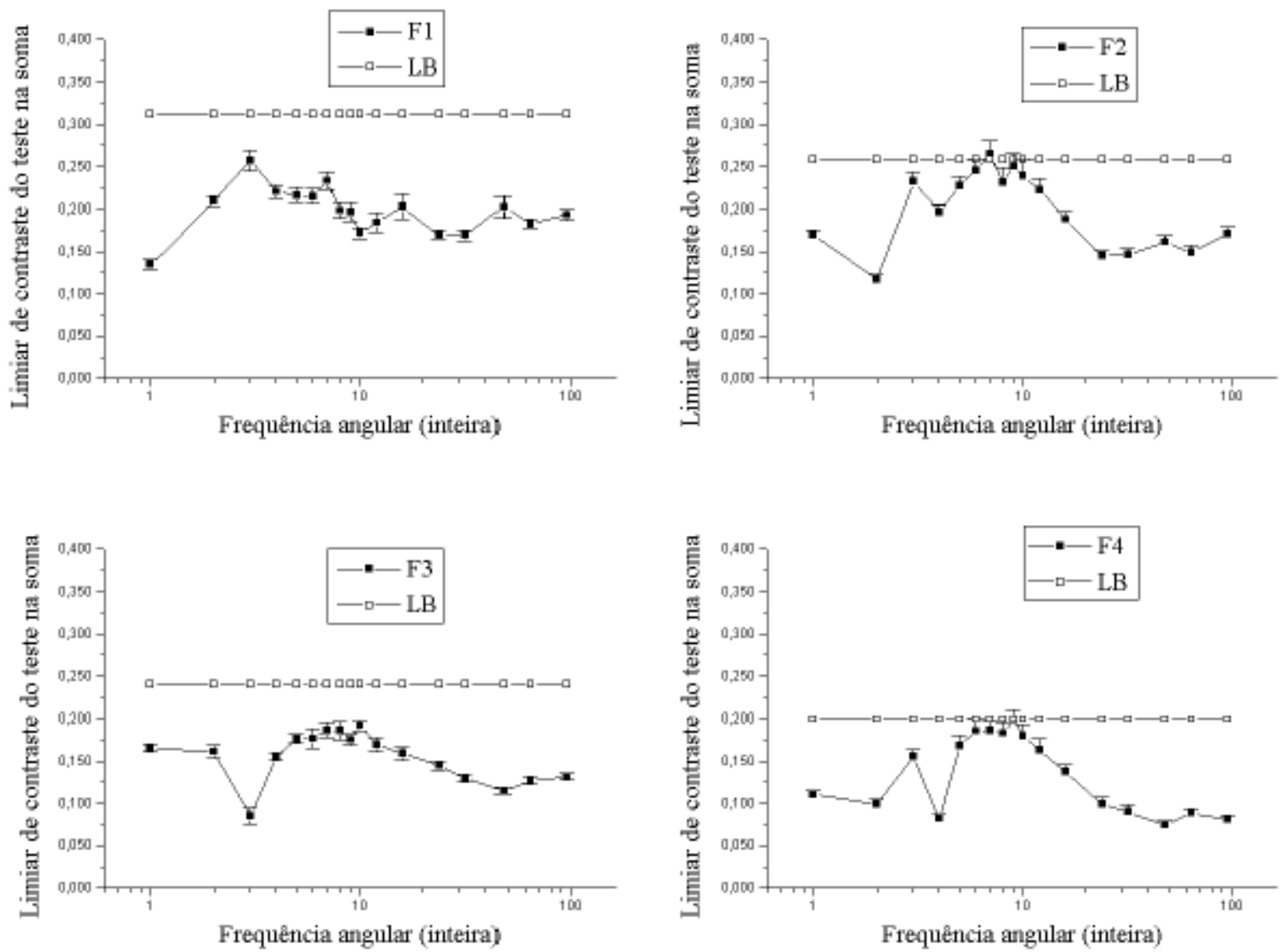

Figura 2. Curvas de resposta ao contraste da freqüência angular de teste de 1 (acima à esquerda), de 2 (acima à direita), de 3 (abaixo à esquerda) e de 4 ciclos $/ 360^{\circ}$ (abaixo à direita), somada às respectivas freqüências angulares de fundo representadas na abscissa. A linha horizontal representa a linha de base (LB), que equivale ao limiar absoluto para detectar a freqüência angular de teste do filtro (1, 2, 3 e 4 ciclos $/ 360^{\circ}$ ) isolada. 
do como referência o limiar absoluto de detecção da freqüência de teste, é o que caracteriza ou não o processo de filtragem.

As razões entre somação e inibição máximas foram da ordem de 1,9; 2,0; 1,9 e 2,1 para os filtros angulares de F1, F2, F3 e F4, respectivamente. Estas razões foram encontradas nas freqüências vizinhas (por exemplo, 3 ciclos no caso do filtro angular F1; 3,0 ciclos no caso do filtro angular F2; 2 ciclos no caso do filtro angular F3 e 5 ciclos no caso do filtro angular F4) indicando claramente efeitos de somação ou filtragem. Em outras palavras, a banda de passagem de cada filtro angular apresentou alta seletividade, o que implicou em somação máxima na freqüência de teste de cada filtro circundada em ambos os lados por efeito de inibição (Figura 2). As razões entre somação e inibição foram calculadas dividindo o limiar de detecção de contraste da freqüência vizinha à freqüência de teste do filtro que sofreu maior inibição pelo limiar de detecção de contraste obtido na freqüência de teste do próprio filtro angular.

Estes resultados são consistentes com a existência de filtragens ou mecanismos de banda estreita operando na faixa de freqüência angular baixa estudada.

\section{Discussão}

Os resultados com filtros de freqüência angular F1, F2, F3 e F4 apresentam somação máxima ou relativa na freqüência de teste de cada filtro angular mensurado. Observa-se ainda uma banda-estreita de passagem ou filtragem bem delimitada para todos os filtros investigados, o que de certa forma mostra uma alta seletividade do SVH para freqüências angulares. Estes resultados são consistentes com os dados de Simas e colaboradores (Simas \& Dodwell, 1990; Simas et al., 1992), que apresentaram somações máximas de limiares de contraste nas freqüências angulares de teste de todos os filtros angulares circundadas por inibições nas freqüências angulares vizinhas as freqüências de teste. Esse efeito foi verificado para todos os filtros estudados.

Um outro aspecto importante é que os nossos resultados com filtros de freqüência angular F1, F2, F3 e F4 também apresentam efeitos de somação secundários para freqüências angulares, quando a freqüência de teste do filtro é somada a freqüência angular de fundo mais baixas e mais altas. Por exemplo, o filtro angular F1 apresentou efeito de somação relativo nas freqüências de fundo mais altas (e.g., 9, 24 e 32 ciclos); por sua vez, o filtro angular F2 apresentou somação relativa na freqüência angular de fundo de 4, 24, 32 e 64 ciclos; enquanto, o filtro angular F3 apresentou somação relativa na freqüência de fundo de 48 ciclos e por último, o filtro angular F4 apresentou efeito de somação relativa em freqüência de fundo mais baixas (2 ciclos) e mais altas (48 ciclos), respectivamente. Estes resultados mostram evidências para interações entre canais de freqüências angulares em coordenadas polares. Resultados nesta direção já foram descritos na literatura para grade senoidal em coordenadas cartesianas (De Valois, 1977; Tolhurst, 1972; Tolhurst \& Barfield, 1978) e também para estímulos de freqüências radiais em coordenadas polares (Santos \& Simas, 2002). Por exemplo, alguns tra- balhos com grade senoidal encontraram casos de sensibilidade aumentada para freqüências espaciais à cerca de uma oitava da freqüência da grade de teste (De Valois, 1977; Tolhurst, 1972; Tolhurst \& Barfield, 1978). Esses autores discutiram que isso constitui evidência para interações entre canais de freqüências espaciais. Alguns resultados também mostram interações entre o fundamental e o terceiro harmônico (Pollen \& Ronner, 1982). Apesar destes resultados serem com padrões cartesianos ao invés de polares, são interessantes na medida em que os nossos resultados para filtros angulares em coordenadas polares também apresentam interação entre canais de freqüência angular de banda estreita. Estes resultados também demostram que os canais para freqüências angulares não são totalmente independentes.

Talvez o pressuposto básico aqui seja a idéia de que, dependendo do filtro espacial (grade senoidal, radial ou angular), mecanismos diferentes interdependentes e intradependentes sejam ativados (efeito de inibição, somação e independência dos canais para freqüências espaciais radiais). Os filtros investigados aqui podem não ser tão perfeitos quanto o filtro ideal (que deixa a quantidade desejada inalterada e reduz todos os outros valores a zero), porém fica a idéia que o sistema de filtragem executado pelo SVH é muito robusto, o que nos leva a crer que a circuitaria visual dispõe de mecanismo para se ajustar a qualquer tipo de padrão espaciais em coordenadas polares ou cartesianas. Desta forma, o sistema visual extrai as características necessárias para reconhecer os detalhes que diferenciam um padrão de outro de acordo com as "suas necessidades". Talvez o objetivo principal do sistema visual seja representar o ambiente visual da maneira mais concreta para a realidade na qual o sistema foi adaptado. Há quem acredite que o sistema visual exista para derivar da imagem a informação de que nós precisamos e não simplesmente para recriar a imagem projetada na retina (Braddick, Campbell, \& Atkinson, 1978).

Em síntese, estes resultados fornecem evidências psicofísicas para a existência de filtros ou canais distintos sintonizados para o processamento de padrões simétricos em coordenadas polares, por exemplo, freqüências angulares baixas. Entretanto, por outro lado, estes resultados sobre filtragem angular não podem provar por si só que o sistema visual utiliza este tipo de filtro para processar e reconhecer objetos ou uma cena visual. Portanto, o fato de o SVH ser sensível a estímulos desta natureza, por si só é um indício forte da importância destes estímulos no processamento visual de forma.

\section{Agradecimento}

Os autores agradecem o apoio financeiro de CNPq (Conselho Nacional de Desenvolvimento Científico e Tecnológico) e FACEPE (Fundação de Amparo à Ciência e Tecnologia do Estado de Pernambuco).

\section{Referências}

Blakemore, C., \& Campbell, F. C. (1969a). Adaptation to spatial stimuli. Journal of Physiology, 200, 11-12. 
Blakemore, C., \& Campbell, F. C. (1969b). On the existence of neurons in the human visual system selectively sensitive to the orientation and size of retinal images. Journal of Physiology, 203, 237-260.

Braddick, O., Campbell, F. W., \& Atkinson, J. (1978). Channels in vision: basic aspects. In R. Held, H. W. Leibowitz, \& H. L. Teuber (Orgs.), Handbook of sensory physiology: perception (Vol. V, pp. 3-38). Nova York: Springer-Verlag.

Bruce, C. J., Desimone, R., \& Gross, C. G. (1981). Visual properties of neurons in a polysensory area in superior temporal sulcus of the macaque. Journal of Neurophysiology, 46, 369-384.

Campbell, F. W., \& Robson, F. G. (1968). Application of the Fourier analysis to the visibility of gratings. Journal of Physiology, 197, 551-566.

Dakin, S. C., \& Hess, R. F. (1997). The spatial mechanisms mediating symmetry perception. Vision Research, 37, 2915-2939.

De Valois, K. K. (1977). Spatial frequency adaptation can enhance contrast sensitivity. Vision Research, 17, 1057-1065.

De Valois, R. L., \& De Valois, K. K. (1988). Spatial vision. Nova York: Oxford University Press.

Desimone, R. (1991). Face-selective cells in the temporal cortex of monkeys. Journal of Cognitive Neuroscience, 3, 1-8.

Desimone, R., \& Schein, S. J. (1987). Visual properties of neurons in area V4 of macaque: sensitivity to stimulus form. Journal of Neurophysiology, 57, 835-867.

Gallant, J. L., Connor, C. E., Rakshit, S., Lewis, J. W., \& van Essen, D. C. (1996). Neural responses to polar, hyperbolic, and cartesian gratings in area V4 of the macaque monkey. Journal of Neurophysiology, 76, 2718-2739.

Gilinski, A. S. (1968). Orientation-specific effects of patterns of adapting light on visual acuity. Journal of the Optical Society of America, 58, 13-18.

Heywood, C. A., Gadotti, A., \& Cowey, A. (1992). Cortical area V4 and its role in the perception of color. Journal of Neuroscience, 12, 4056-4065.

Kulikowski, J. J., \& King-Smith, P. E. (1973). Spatial arrangement of line, edge and grating detectors released by sub-threshold summation. Vision Research, 13, 1455-1478.

Maffei, L., \& Fiorentini, A. (1973). The visual cortex as a spatial frequency analyzer. Vision Research, 13, 1255-1267.

Manahilov, V., \& Simpson, W. A. (2001). Energy model for contrast detection: spatial-frequency and orientation selectivity in grating summation. Vision Research, 41, 1547-1560.

Menees, S. M. (1998). The effect of spatial frequency adaptation on the latency of spatial contrast detection. Vision Research, 38, 3933-3942.

Merigan, W. H. (1996). Basic visual capabilities and shape discrimination after lesions of extrastriate area V4 in macaques. Visual Neuroscience, 13, 51-60.

Olds, E. S., \& Engel, S. A. (1998). Linearity across spatial frequency in object recognition. Vision Research, 38, 2109-2118.

Pollen, D. A., \& Ronner, S. F. (1982). Spatial computation performed by simple and complex cells in the visual cortex of the cat. Vision Research, 22, 101-118.

Sachs, M. B., Nachmias, J., \& Robson, J. G. (1971). Spatial frequency channels in human vision. Journal of the Optical Society of America, 61, 1176-1186.

Santos, N. A., \& Simas, M. L. B. (2001a). Função de sensibilidade ao contraste: indicador da percepção visual da forma e da resolução espacial. Psicologia: Reflexão e Crítica, 14(3), 589-597.
Santos, N. A., \& Simas, M. L. B. (2001b). Percepção e processamento visual da forma: discutindo modelos teóricos atuais. Psicologia: Reflexão \& Crítica, 14(1), 151-160.

Santos, N. A., \& Simas, M. L. B. (2002). Percepção e processamento visual da forma em humanos: filtros de freqüências radiais de 1 e 4 cpg. Psicologia: Reflexão e Crítica, 15(2), 383-391.

Santos, N. A., Simas, M. L. B., \& Nogueira, R. M. T. B. L. (2004). Comparison of angular frequency contrast sensitivity in young and older adults. Brazilian Journal of Medical and Biological Research, 37, 375-378.

Simas, M. L. B., \& Dodwell, P. C. (1990). Angular frequency filtering: a basis for pattern decomposition. Spatial Vision, 5, 59-74.

Simas, M. L. B., Frutuoso, J. T., \& Vieira, F. M. (1992). Inhibitory sidebands in multiple angular filters in the human visual system. Brazilian Journal of Medical and Biologocal Research, 25, 919-923.

Simas, M. L. B., \& Santos, N. A. (2002a). Contrast sensitivity to radial frequencies modulated by Jn and jn Bessel profiles. Brazilian Journal of Medical and Biological Research, 35, 1357-1366.

Simas, M. L. B., \& Santos, N. A. (2002b). Narrow-band 1, 2, 3, 4, 8, 16 and 24 cycles $/ 360^{\circ}$ angular frequency filters. Brazilian Journal of Medical and Biological Research, 35, 243-253.

Tolhurst, D. J. (1972). Adaptation to square-wave gratings: inhibition between spatial frequency channels in the human visual system. Journal of Physiology, 226, 231-248.

Tolhurst, D. J., \& Barfield, L. P. (1978). Interactions between spatial frequency channels. Vision Research, 18, 851-858.

van Essen, D. C., Anderson, C. H., \& Felleman, D. J. (1992). Information processing in the primate visual system: an integrated systems perspective. Science, 255, 419-423.

Wetherill, G. B., \& Levitt, H. (1965). Sequential estimation of points on a psychometric function. The British Journal of Mathematical and Statistical Psychology, 48, 1-10.

Wilkinson, F., James, T. W., Wilson, H. R., Gati, J. S., Menon, E. S., \& Goodale, M. A. (2000). An fMRI study of the selective activation of human extrastriate form vision areas by radial and concentric gratings. Current Biology, 10, 1455-1458.

Wilkinson, F., Wilson, H. R., \& Habak, C. (1998). Detection and recognition of radial frequency patterns. Vision Research, 38, 3555-3568.

Wilson, H. R., Levi, D., Maffei, L., Rovamo, J., \& De Valois, R. (1990). The perception of form: retina to striate cortex. In S. W. Spillmann \& J. S. Werner (Orgs.), Visual perception: the neurophysiological foundation (pp. 231-271). Nova York: Academic.

Wilson, H. R., \& Wilkinson, F. (1997). Evolving concepts of spatial channels in vision: from independence to nonlinear interactions. Perception, 26, 939-960.

Wilson, H. R., \& Wilkinson, F. (1998). Detection of global structure in glass patterns: implications for form vision. Vision Research, 38, 2933-2947.

Wilson, R. W., Wilkinson, F., \& Asaad, W. (1997). Concentric orientation summation in human form vision. Vision Research, 37, 2325-2330.

Young, M. P. (1992). Objective analysis of topological organization of the primate cortical visual system. Nature, 358, 152-155.

Natanael Antonio dos Santos, doutor em Psicologia (Neurociências e Comportamento) pela Universidade de São Paulo, é professor adjunto no Mestrado de Psicologia, Universidade Federal da Paraíba. Endereço para correspondência: Departamento de Psicologia, CCHLA-UFPB; Campus I, Cidade Universitária; Conjunto Castelo Branco; João Pessoa, PB; CEP 58051-900. Tel.: (83) 3216-7006. Fax: (83) 3216-7064. Email: natanael.santos@uol.com.brou natanael@cchla.ufpb.br

Maria Lúcia de Bustamante Simas, doutora em Psicologia pela Queen’s University, Ontario, Canada, é professora adjunta no Departamento de Psicologia, Universidade Federal de Pernambuco e pesquisadora do CNPq. Renata Maria Toscano Barreto Lyra Nogueira é mestranda em Psicologia, Universidade Federal da Paraíba. 